\title{
Fizeau interferometric imaging of Io volcanism with LBTI/LMIRcam
}

\author{
J. M. Leisenring ${ }^{a}$, P. M. Hinz ${ }^{a}$, M. F. Skrutskie ${ }^{b}$, A. Skemer ${ }^{a}$, C. E. Woodward ${ }^{c}$, C. Veillet ${ }^{d}$,

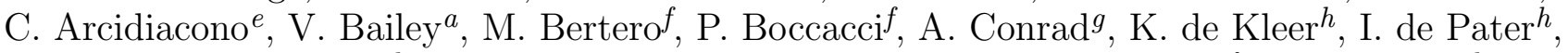

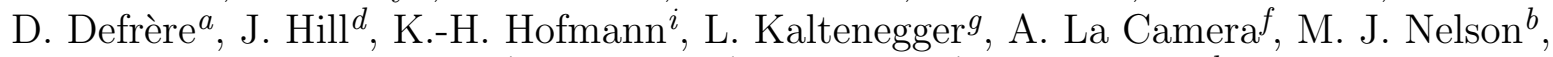 \\ D. Schertl ${ }^{i}$, J. Spencer ${ }^{j}$, G. Weigelt ${ }^{i}$, J. C. Wilson ${ }^{b}$ \\ ${ }^{a}$ Steward Observatory, University of Arizona, 933 N. Cherry Ave, Tucson, AZ 85721, USA; \\ ${ }^{b}$ University of Virginia, 530 McCormick Rd, Charlottesville, VA 22904, USA; \\ ${ }^{c}$ University of Minnesota, 116 Church St SE, Minneapolis, MN 55455, USA; \\ ${ }^{d}$ LBT Observatory, University of Arizona, 933 N. Cherry Ave, Tucson, AZ 85721, USA; \\ ${ }^{e} \mathrm{INAF}$ - Osservatorio Astronomico di Bologna, Via Ranzani 1, I-40127 Bologna, Italy; \\ fDIBRIS, Genova University, Via Dodecaneso 35, 16146 Genova, Italy; \\ ${ }^{g}$ Max-Planck-Institut für Astronomie, Königstuhl 17, 69117 Heidelberg, Germany; \\ ${ }^{h}$ Astronomy Department, 601 Campbell Hall, Univ. of California, Berkeley, CA 94720, USA; \\ ${ }^{i}$ Max-Planck-Institut für Radioastronomie, Auf dem Hügel 69, 53121 Bonn, Germany; \\ ${ }^{j}$ Southwest Research Institute, 1050 Walnut St, Suite 300, Boulder, CO 80302
}

\begin{abstract}
The Large Binocular Telescope (LBT) houses two 8.4-meter mirrors separated by 14.4 meters on a common mount. Coherent combination of these two AO-corrected apertures via the LBT Interferometer (LBTI) produces Fizeau interferometric images with a spatial resolution equivalent to that of a 22.8 -meter telescope and the lightgathering power of single 11.8-meter mirror. Capitalizing on these unique capabilities, we used LBTI/LMIRcam to image thermal radiation from volcanic activity on the surface of Io at $M$-Band $(4.8 \mu \mathrm{m})$ over a range of parallactic angles. At the distance of Io, the $M$-Band resolution of the interferometric baseline corresponds to a physical distance of $\sim 135 \mathrm{~km}$, enabling high-resolution monitoring of Io volcanism such as flares and outbursts inaccessible from other ground-based telescopes operating in this wavelength regime. Two deconvolution routines are used to recover the full spatial resolution of the combined images, resolving at least sixteen known volcanic hot spots. Coupling these observations with advanced image reconstruction algorithms demonstrates the versatility of Fizeau interferometry and realizes the LBT as the first in a series of extremely large telescopes.
\end{abstract}

Keywords: Infrared, Fizeau, Interferometry, Large Binocular Telescope, LMIRcam, Deconvolution, Io, Volcano

\section{INTRODUCTION}

With two 8.4-meter situated mirrors on a common mount, the Large Binocular Telescope (LBT) is positioned as the first in a class of extremely large telescopes (ELT). While the two apertures are routinely operated independently of one another, each using their own state-of-the-art AO system, interferometric combination of the two telescope beams clearly differentiates the LBT from traditional 8-meter class telescopes. The NASAfunded LBT Interferometer (LBTI) currently serves as the LBT's primary beam combiner, ${ }^{1,2}$ ultimately taking advantage of the high-performance $\mathrm{AO}$ system $^{3,4}$ in order to produce Fizeau interferometric images at the instrument's combined focal plane.

The Fizeau PSF can be described by an Airy disc of a single 8.4-meter aperture along the vertical/altitude axis and the product of that Airy disc with a two-slit diffraction pattern along the horizontal/azimuth axis. The interferometric axis provides a resolution equivalent to a 22.8 -meter telescope for a single image. As the field of view rotates for an object observed by an Alt-Az telescope, a range of parallactic angles (PA) becomes accessible

Optical and Infrared Interferometry IV, edited by Jayadev K. Rajagopal, Michelle J. Creech-Eakman,

Fabien Malbet, Proc. of SPIE Vol. 9146, 91462S • ( 2014 SPIE • CCC code: 0277-786X/14/\$18

doi: $10.1117 / 12.2057290$

Proc. of SPIE Vol. 9146 91462S-1 
Table 1. Io Observations - 2013-12-24

\begin{tabular}{|c|ccc|ccc|}
\hline $\begin{array}{c}\text { Nod } \\
\text { Number }\end{array}$ & $\begin{array}{c}\text { Start Time } \\
(\mathbf{U T})\end{array}$ & $\begin{array}{c}\text { RA } \\
(\mathbf{J 2 0 0 0})\end{array}$ & $\begin{array}{c}\text { Dec } \\
(\mathbf{J 2 0 0 0})\end{array}$ & $\begin{array}{c}\text { \# Images } \\
\text { Combined }\end{array}$ & $\begin{array}{c}\text { Mean Para. } \\
\text { Angle }\end{array}$ & $\begin{array}{c}\text { Io Relative } \\
\text { Orbital Rot. }\end{array}$ \\
\hline 1 & $07: 53$ & $7 \mathrm{~h} 13 \mathrm{~m} 17.7 \mathrm{~s}$ & $22 \mathrm{~d} 28 \mathrm{~m} 33.9 \mathrm{~s}$ & 150 & -30.0 & $0.0^{\circ}$ \\
2 & $07: 59$ & $7 \mathrm{~h} 13 \mathrm{~m} 17.6 \mathrm{~s}$ & $22 \mathrm{~d} 28 \mathrm{~m} 34.2 \mathrm{~s}$ & 138 & -22.2 & $0.8^{\circ}$ \\
3 & $08: 06$ & $7 \mathrm{~h} 13 \mathrm{~m} 17.6 \mathrm{~s}$ & $22 \mathrm{~d} 28 \mathrm{~m} 34.4 \mathrm{~s}$ & 70 & -15.9 & $1.8^{\circ}$ \\
4 & $08: 13$ & $7 \mathrm{~h} 13 \mathrm{~m} 17.5 \mathrm{~s}$ & $22 \mathrm{~d} 28 \mathrm{~m} 34.7 \mathrm{~s}$ & 79 & -7.5 & $2.9^{\circ}$ \\
5 & $08: 24$ & $7 \mathrm{~h} 13 \mathrm{~m} 17.3 \mathrm{~s}$ & $22 \mathrm{~d} 28 \mathrm{~m} 35.0 \mathrm{~s}$ & 94 & 4.1 & $4.3^{\circ}$ \\
6 & $08: 35$ & $7 \mathrm{~h} 13 \mathrm{~m} 17.2 \mathrm{~s}$ & $22 \mathrm{~d} 28 \mathrm{~m} 35.3 \mathrm{~s}$ & 104 & 16.3 & $6.0^{\circ}$ \\
7 & $08: 47$ & $7 \mathrm{~h} 13 \mathrm{~m} 17.0 \mathrm{~s}$ & $22 \mathrm{~d} 28 \mathrm{~m} 35.8 \mathrm{~s}$ & 108 & 29.1 & $7.7^{\circ}$ \\
\hline
\end{tabular}

along the high-resolution axis, ultimately recovering the diffraction-limited spatial resolution of a full 22.8-meter circular aperture.

Operating primarily within the $L(3.6 \mu \mathrm{m})$ and $M(4.8 \mu \mathrm{m})$ atmospheric windows at the LBTI's combined focus, LMIRcam ${ }^{5,6}$ has seen regular operations where both telescope beams have been incoherently overlapped to produce high-Strehl images. ${ }^{7}$ In order to obtain interferometric fringes, path lengths are adjusted such that the overlapped beams are coherently combined to produce the characteristic diffraction pattern along the direction of the two-telescope baseline.

Without closed-loop adjustments, the interference pattern oscillates around the central fringe due to small changes in the path-length difference from instrumental vibration and atmospheric disturbance. If the characteristic timescale of these oscillations is sufficiently large, fast frame acquisition can capture images where the path-length difference is near zero. Known as 'lucky Fizeau,' the frame quality is ranked during post-processing and the best frames retained for image reconstruction. LBTI's PhaseCam will ultimately carry out closed-loop adjustments of tip-tilt and phase variations to stabilize the PSF. ${ }^{8}$

\section{OBSERVATIONS AND DATA REDUCTION}

Around the time of its January 2014 opposition, Jupiter was near maximum northerly declination, providing high-airmass observations from Mt. Graham and, thus, a relatively large range of parallactic angles over a short period during transit of the meridian. Folding in Io's 42.5-hour tidally-locked orbit constrained the observing window on a given night such that Io's angular separation from Jupiter was sufficiently far that Jupiter's scattered light did not interfere with $\mathrm{AO}$ operations or science observations and that the rotation of Io with respect to the observer was minimized over the observing period.

We acquired LMIRcam $M$-Band $(4.6-5.0 \mu \mathrm{m})$ interferometric images of Io during UT 2013 December 24. Because phase stabilization was not fully operational, PhaseCam only supplied tip-tilt corrections to keep the two telescope beams overlapped. Coherent combination was accomplished by manually adjusting the path-length difference in open loop while observing the PSF fringes. Due to the short timescales of the phase variations, the science detector frame size was reduced to a $256 \times 256$ subarray, accommodating a shorter readout time of $17 \mathrm{~ms}$ for capturing 'lucky fringes.'

A total of seven nod sequences were acquired (Table 1), consisting of 3000 science frames per nod interleaved with 1000 off-nod background frames. Each science frame was sky- and dark-subtracted using the mediancombination of corresponding background frames. Reference pixels located at the top and bottom of each frame provided a means of removing the relative offsets between readout channels due to time-dependent voltage drifts. A master bad pixel mask was then generated by median combining background-subtracted frames for each nod sequence and flagging locally-deviant pixels. Those bad pixels were subsequently fixed in each frame using the median of adjacent pixels.

We acquired a sequence of 500 frames of the reference star HD 78141 and reduced the data in a similar manner as the Io science data. The reduced images were cross-correlated with a theoretical monochromatic $(4.8 \mu \mathrm{m})$ PSF to select those instances when the fringe pattern was in the optimal position. In addition to generating 

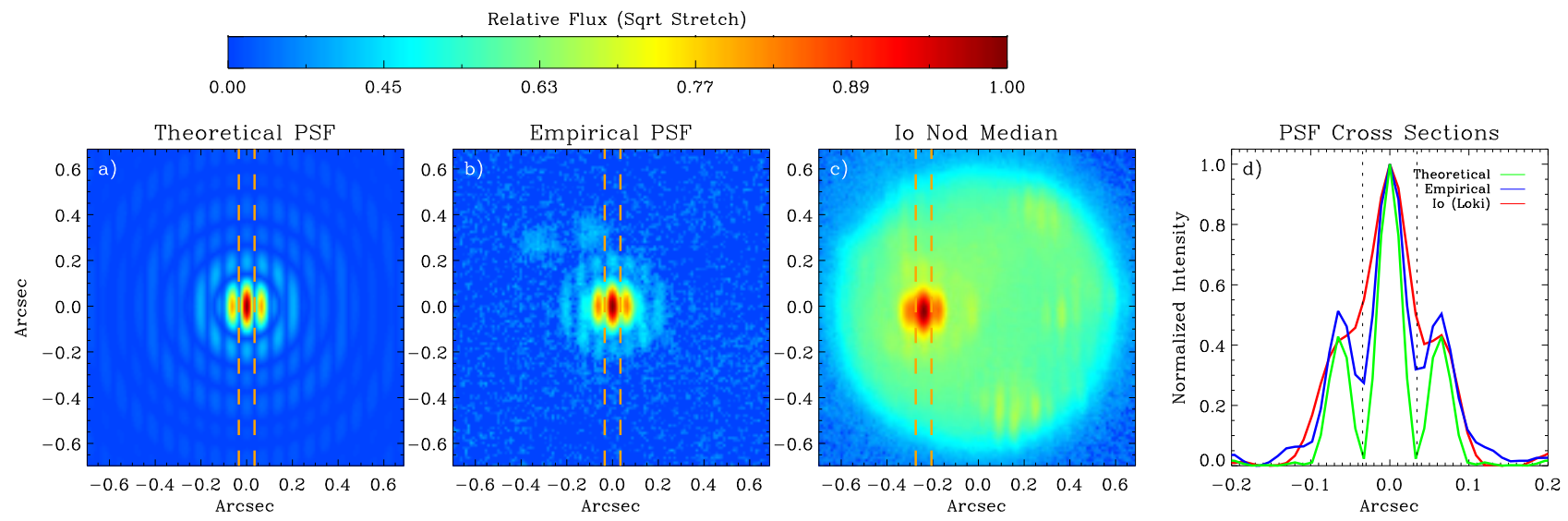

Figure 1. a) Image of theoretical monochromatic PSF displayed in a square root stretch. Vertical dashed lines indicate location of the interferometric null positions for a wavelength of $\lambda=4.8 \mu \mathrm{m}$. b) Observed PSF of the star HD 78141 using the top $10 \%$ of frames out of a sequence of 500 . The two faint spots in the upper left are due to internal reflections of the two telescope beams at the cryogenic beamsplitter. c) A nod sequence of Io after combining the top 5\% of correlated frames. d) Cross-sectional cuts through the center of the PSFs along the horizontal with the maximum value normalized to 1.0. The background surrounding the Loki PSF has been subtracted by fitting a polynomial to Io's surface continuum flux. Loki's PSF profile shows a low visibility along the interferometric axis, suggesting that the hot spot is partially resolved.

correlation coefficients, the cross-correlation routine sampled the relative positions of each frame with sub-pixel accuracy. The final empirical reference PSF was generated by selecting the top $10 \%$ of frames with the highest correlation and median-combining the shifted images. Increasing the number of frames substantially reduced the final PSF visibility while gaining little benefit in $\mathrm{S} / \mathrm{N}$, while fewer frames suffered from low $\mathrm{S} / \mathrm{N}$.

Reduced images of Io were ranked in a similar fashion as the reference PSF, instead using the empirical PSF built from the reference star as opposed to the theoretical PSF. A single hot spot on Io corresponding to the location of the volcano Loki appears prominently at $M$-Band. Even though the volcanic hot spot is slightly resolved along the two-telescope baseline, cross-correlation of Loki with the reference PSF supplies both a ranking of the optical path difference errors as well as frame-to-frame sub-pixel registration. For each nod, only the top $5 \%$ of images were chosen to ensure high visibility along with high $\mathrm{S} / \mathrm{N}$. In order to further remove rotational blurring at the frame edges, those images with parallactic angles greater than $\pm 1^{\circ}$ from the average were discarded, retaining less than $5 \%$ for periods of fast PA rotation. Remaining images were de-rotated by their parallactic angle such that sky North is positioned in the $+\mathrm{y}$ direction and subsequently median-combined to produce the final image for a given nod.

Figure 1 compares the LBT's theoretical PSF, observed stellar PSF, and a final nod image of Io. The fringes of the theoretical PSF are much sharper than observed due to its monochromatic nature, whereas the empirical PSF convolves the entire wavelength range of the M-Band filter. The vertical dotted lines indicate the location of the first interferometric nulls, showing strong agreement between the theoretical and observed PSFs, albeit with lower visibility for the observed PSF due to the lucky-Fizeau nature of the observations. The fringes of Io's bright source Loki show significantly lower visibility than the reference PSF, suggesting the volcanic hotspot is resolved along the high-resolution direction.

Displayed in Figure 2, the seven nod positions have been de-rotated according to their parallactic angle such that sky North is up. As a comparison to the resolution of the 8.4-meter aperture, the figure's last panel shows the average of all incoherent frames. In addition to Loki, multiple hotspots are apparent on interferometric images of Io's surface, which we attempt to separate spatially through deconvolution and correlate with known volcanic activity. 


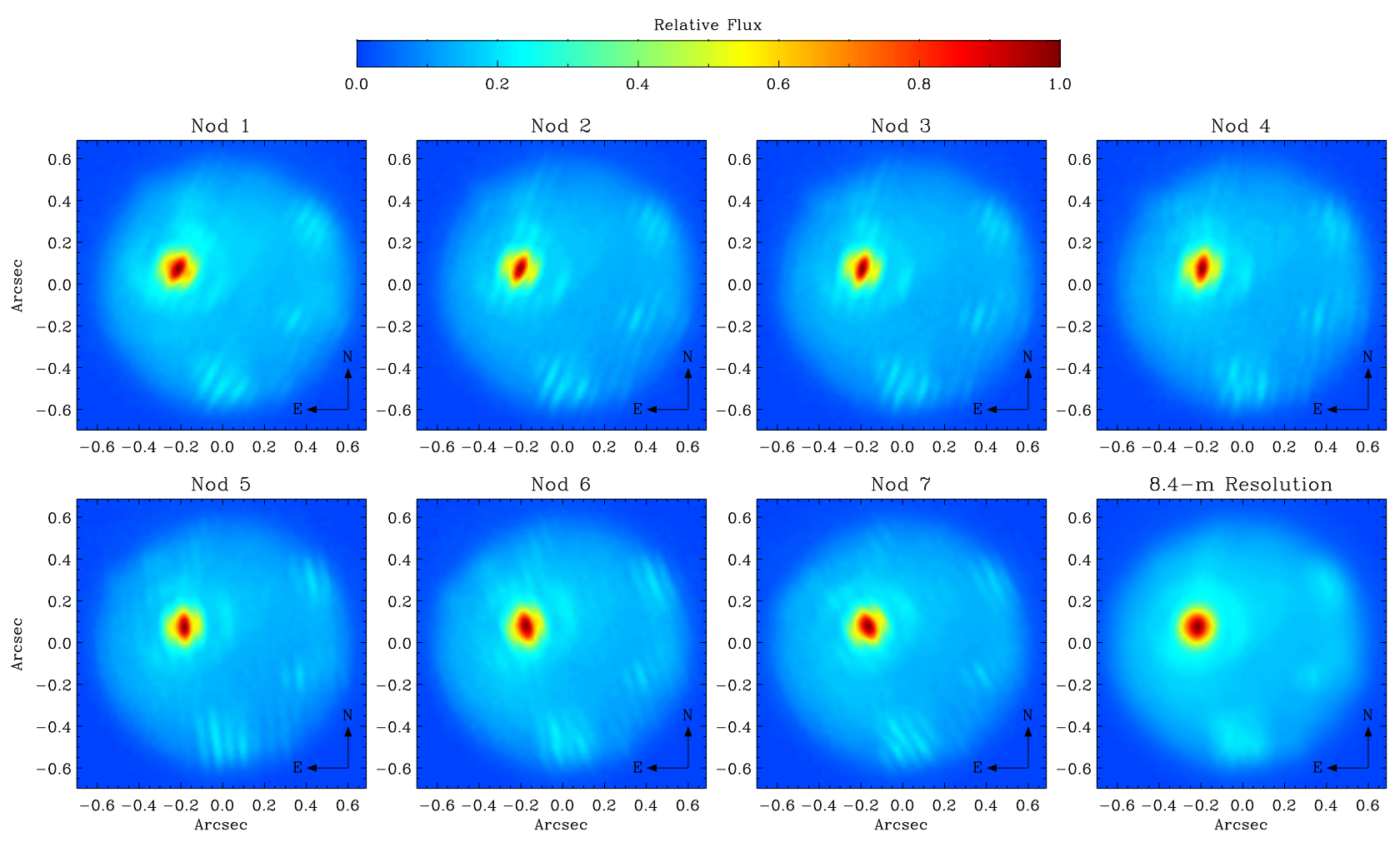

Figure 2. The seven nods used for reconstruction of the final high-resolution image after de-rotation by PA. The lowerright panel shows the sum of the low-visibility frames, roughly equivalent to a single 8.4-meter aperture. Each nod image has been rotated by the mean parallactic angle of its image stack such that sky North points upward and East is to the left. Comparing the first and last nod sequences reveals the movement of Loki on the projected surface of Io as the moon rotates in its tidally-locked orbit around Jupiter.

\section{IMAGE RECONSTRUCTION}

Recovering a high-resolution image of the scientific target observed with a Fizeau interferometer is a relatively recent problem largely stimulated by the design of the existing LBTI and the future LINC-NIRVANA. ${ }^{9,10}$ The uv coverage of these interferometers consists of a central disc, corresponding to a single 8.4-meter mirror, flanked by two identical discs produced by interferometry. Due to this structure, a set of images taken at different parallactic angles over a range of approximately $120^{\circ}$ produces a uv coverage corresponding to a 22.8-meter telescope or 44 mas at $\lambda=4.8 \mu \mathrm{m}$. At a distance of $4.23 \mathrm{AU}$, this resolution corresponds to $135 \mathrm{~km}$ on the surface of Io. However, in the case of our Io observations, the PA coverage of only $59^{\circ}$ leads to an incomplete uv coverage as shown in Figure 3.

One possible approach to image reconstruction consists in a centered co-adding of the images and of the corresponding PSFs. In this way the problem is reduced to a classic single image deconvolution and one can use the many methods produced for this problem. Another approach consists in designing specific deconvolution methods which have as an input the different images and PSFs. The methods combine these images in some suitable way to produce a single reconstructed image of the scientific target.

Examples of these two deconvolution methods were employed to reconstruct a high-resolution image and validate the identification of bright sources on the surface of Io. Overall, the final reconstructions of Io are degraded by the following limitations: (1) The point spread function measured with a single calibrator star is not identical to the PSF during the Io observations because the AO performance is different during the target and single-star calibrator observation. (2) The uv coverage of the Io observations is not complete because the range 


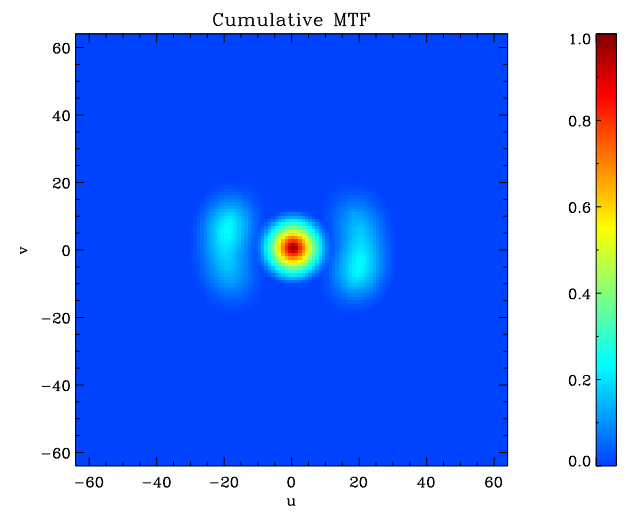

Figure 3. uv coverage of Io observations.

of parallactic Io angles is only $59^{\circ}\left(\sim 120^{\circ}\right.$ would be optimal). (3) The Io rotation during the observations causes some blurring during the image reconstruction. Under these limitations, we find that both methods produce very similar results.

\subsection{Building-Block Method}

The Building-Block (BB) method ${ }^{11,12}$ is an iterative multi-frame deconvolution method. BB reconstruction was performed using the software package available at http://www.mpifr-bonn.mpg.de/295304/linc_drs. As a first step, the reduced science images are de-rotated to correct the parallactic angle changes, centered, and co-added. The result produces target images that are the convolution of the target intensity distribution with a co-added sum PSF. Because the LBTI PSFs are rotated against each other, the coadded PSF is dominated by a bright, almost diffraction-limited core, which appears where the central fringes of all individual rotated point source interferograms cross each other. In an iterative process, Delta functions or clusters of Delta function

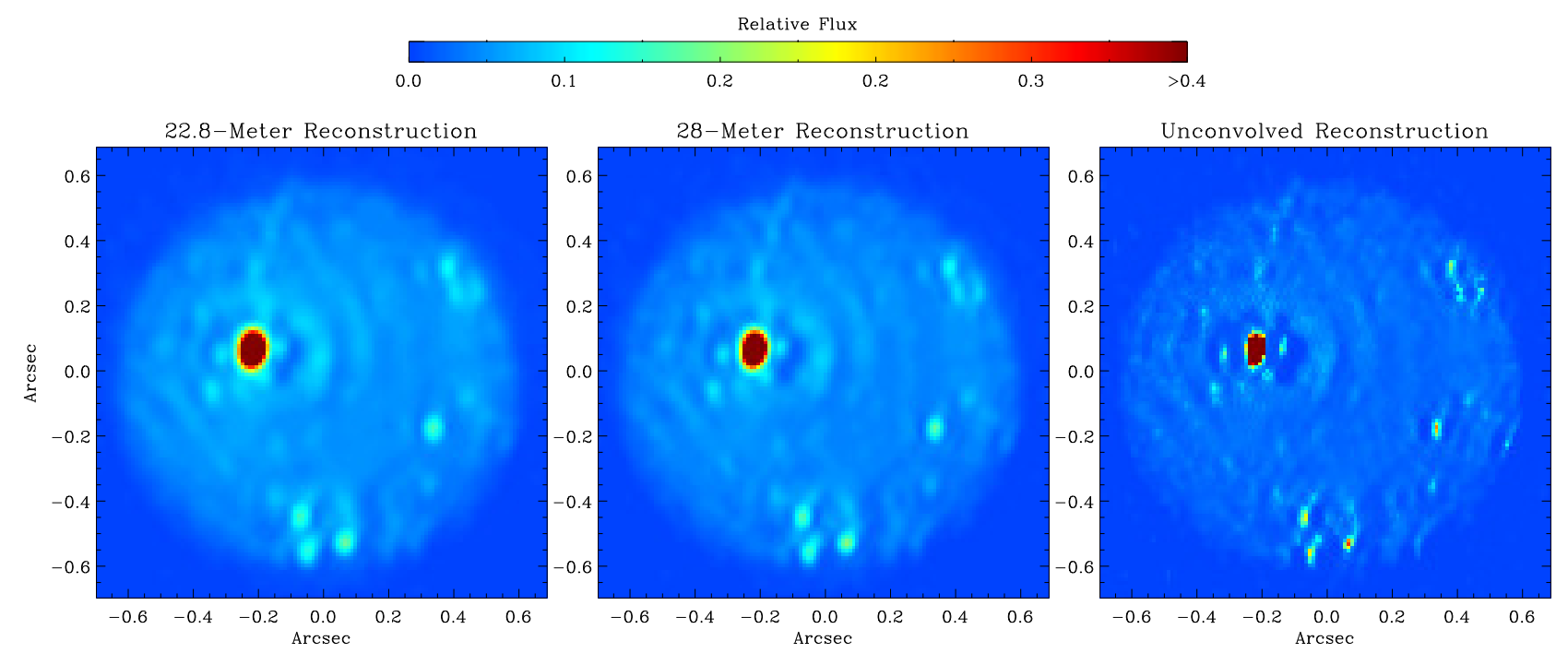

Figure 4. The Building-Block reconstructions of Io calculated with resolutions corresponding to the diffraction-limited resolutions of a 22.8-meter telescope (left), a 28-meter telescope (middle), and the unconvolved reconstruction (right). The bright volcano Loki and about 15 additional point-like objects are visible in the reconstructed image. Loki is not point-like but extended and elongated. Scaling has been adjusted to reveal faint sources. 
(building blocks) are iteratively added to the instantaneous reconstruction in such a way that the distance between the reconstruction and the observations is minimized.

The left-most image presented in Figure 4 shows a diffraction-limited Building-Block reconstruction of Io with a resolution equal to that of a telescope with a circular diameter of 22.8 meters. This resolution is obtained by convolving the original BB reconstruction (Figure 4, right) with the theoretical PSF of a circular 22.8-meter pupil. The brightest volcano Loki is surrounded by a patchy dark ring and a bright diffraction ring, which are most likely deconvolution artifacts. For comparison, the middle panel of Figure 4 also shows a reconstruction calculated with higher resolution (so-called super-resolution) of a 28-meter pupil. The higher angular resolution can lead to more noise or artifacts, for example, deeper dark rings or holes around bright sources. Experiments with computer-simulated data to study this ringing effect suggest that this phenomenom can be caused by errors of the measured PSF and incomplete uv coverage.

\subsection{Multiple-Image Richardson-Lucy Method}

Reconstruction using a variant of the Richardson-Lucy algorithm was performed with an implementation in the software package AIRY v6.0, ${ }^{13,14}$ freely available at http://www.airyproject.eu. The basic method extends on the Richardson-Lucy algorithm to the case of multiple images of the same target (Multiple-image Richardson-Lucy; MRL). ${ }^{15}$ It contains properties similar to those of the standard RL algorithms in that it produces nonnegative reconstructions with a total flux which is the average flux of the interferometric images. The algorithm also accounts for the backgrounds of the different images, removing the background from the restored image. When applied to pre-processed images that have already had their backgrounds removed, such as in the present case, the addition of a small constant is recommended to prevent division by zero. Since the field-of-view of the Io images used for deconvolution was restricted, a modification of the RL algorithm was employed that included a boundary effect correction (BEC). ${ }^{16}$

The left image of Figure 5 shows the MRL+BEC result after 300 iterations. As with the Building Block method, significant ringing is observed around each bright spot, likely due to imperfect knowledge of the PSF, rotation of Io, and/or incomplete uv coverage. The spots are elongated in the vertical direction because parallactic angle coverage ranges between $-30^{\circ}$ and $29^{\circ}$.

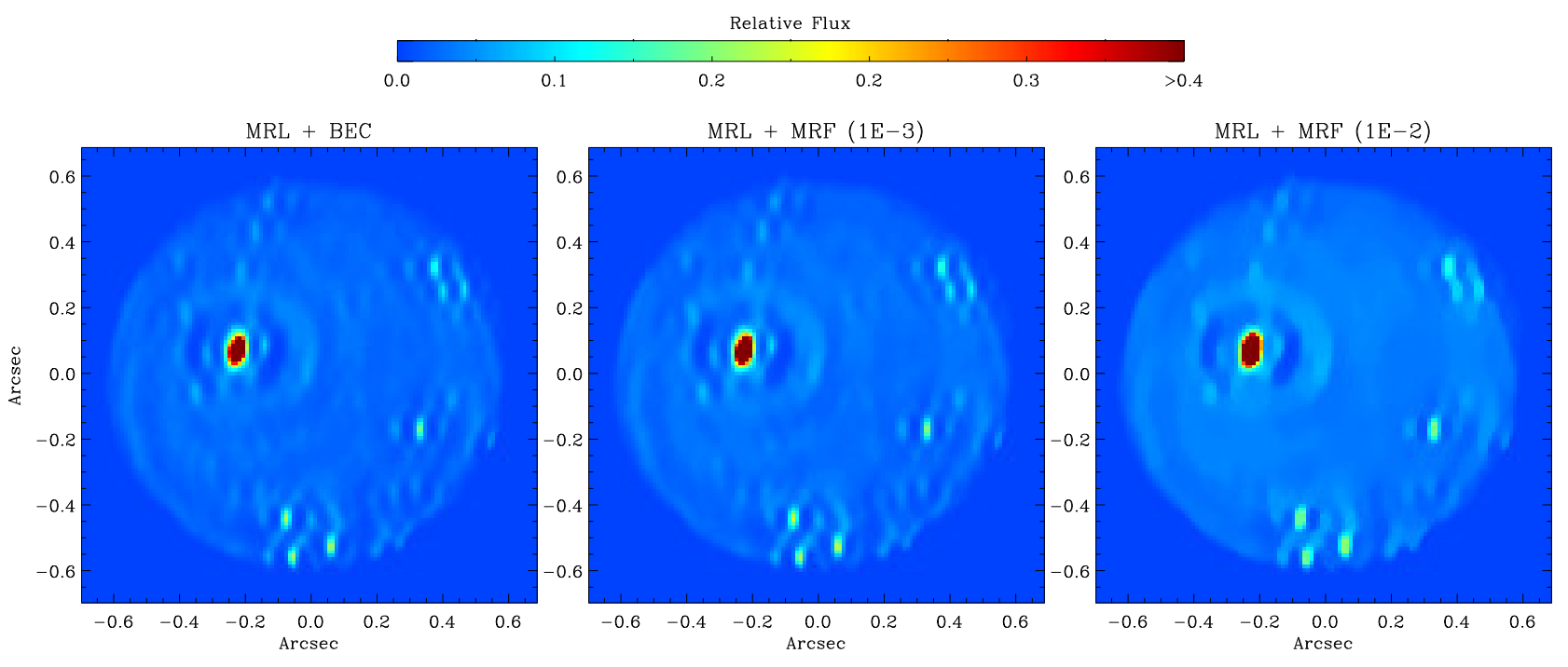

Figure 5. Results of reconstructions obtained with the multiple-image Richardson-Lucy method, including boundary effect corrections in the left panel. Two MRF reconstructions with regularization parameters of $10^{-3}$ and $10^{-2}$ are shown in the middle and right panels, respectively. Scaling is the same as in Figure 4. Unlike the first two images in Figure 4, these results have not been convolved with a PSF. 

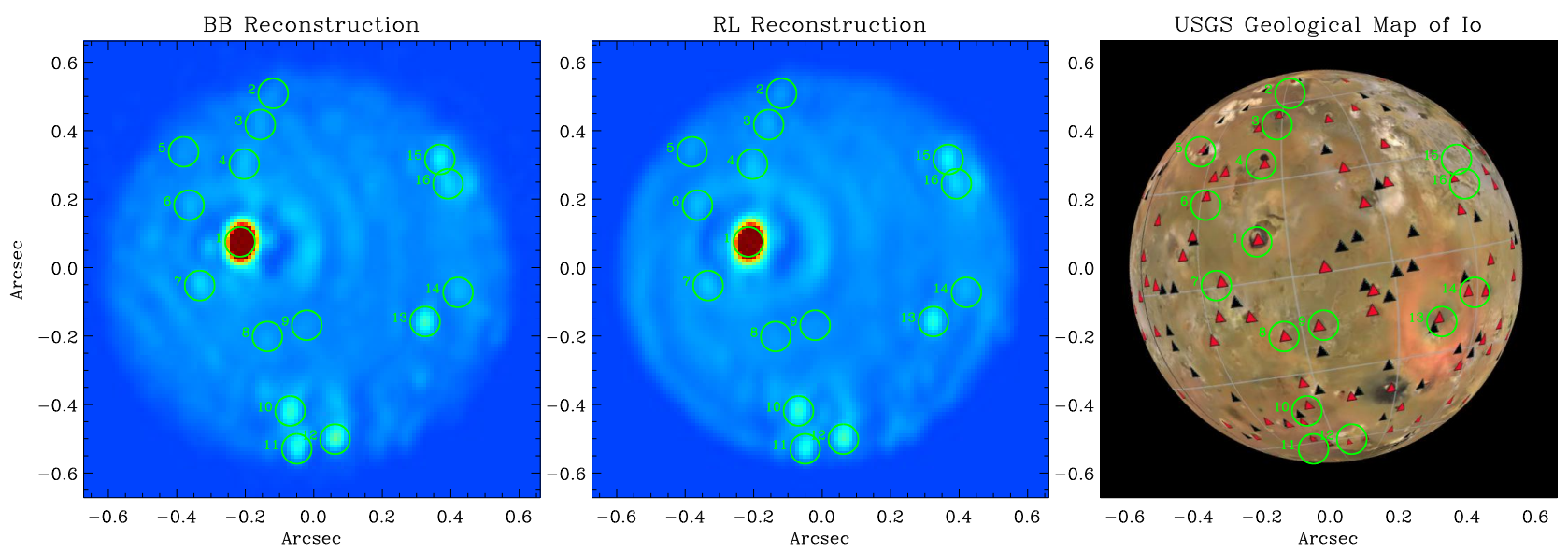

Figure 6. Building-Block and Multiple-image Richardson-Lucy image reconstructions (left and middle, respectively) convolved with the PSF of a 22.8-meter circular pupil. The image on the right is a projection of the USGS Geological Map of Io with known hot spots indicated with a red triangle. Green circles indicate the bright sources identified in either reconstruction at $5 \sigma$ that also correlate with known hot spots.

Because the previous image is affected by a very pronounced ringing effect due to the presence of bright sources over a smooth background (Io surface), a Markov Random Field (MRF) regularization algorithm was also implemented during reconstruction. ${ }^{17,18}$ The use of this algorithm requires an accurate tuning of a parameter called 'regularization parameter,' which has not previously been performed. Two different reconstructions are shown in the middle and right panels of Figure 5, with the regularization parameter set to $10^{-3}$ and $10^{-2}$, respectively. These preliminary results show a reduction in the ringing artifacts and underlying noise, but may also conceal faint sources.

\section{SOURCE IDENTIFICATION}

Both reconstruction techniques produce similar results with point sources consisting of an elongated core in the vertical axis and two side-lobe structures along the horizontal direction: artifacts of the incomplete uv coverage coupled with differential movement of the sources on Io's projected surface. Even with these limitations, there are clear advantages over traditional observations with an AO-corrected 8.4-meter telescope.

Figure 6 shows sixteen sources detected at a 5- $\sigma$ confidence. Respectively, the left and middle images display the BB and MRL reconstructions convolved with a PSF equivalent to a single 22.8-meter circular aperture. The

Table 2. Identified Sources

\begin{tabular}{|cc|cc|cc|cc|cc|cc|}
\hline & Source & \multicolumn{2}{|c|}{ BB } & \multicolumn{2}{c|}{ MRL } & \multicolumn{3}{c|}{ Source } & \multicolumn{2}{c|}{ BB } & \multicolumn{2}{c|}{ MRL } \\
$\#$ & Name & Flux $^{a}$ & S/N & Flux $^{a}$ & S/N & $\#$ & Name & Flux $^{a}$ & S/N & Flux $^{a}$ & S/N \\
\hline 1 & Loki & 1.000 & 32.4 & 1.000 & 33.1 & 9 & Gibil & 0.006 & 8.1 & 0.003 & 4.4 \\
2 & Vivasvant & 0.007 & 3.3 & 0.010 & 5.0 & 10 & Rarog & 0.040 & 10.6 & 0.041 & 11.6 \\
3 & Dazhbog & 0.006 & 4.8 & 0.010 & 7.6 & 11 & Heno & 0.051 & 12.7 & 0.049 & 12.2 \\
4 & Amaterasu & 0.014 & 7.2 & 0.016 & 7.2 & 12 & Lerna & 0.073 & 21.3 & 0.067 & 18.6 \\
5 & Surt & 0.009 & 7.8 & 0.010 & 8.5 & 13 & Pele & 0.054 & 16.4 & 0.047 & 15.9 \\
6 & Fuchi & 0.014 & 10.1 & 0.019 & 10.2 & 14 & Pillan & 0.013 & 13.3 & 0.008 & 7.4 \\
7 & Tol-Ava & 0.022 & 15.0 & 0.010 & 5.4 & 15 & $?$ & 0.034 & 12.9 & 0.038 & 12.5 \\
8 & Mihir & 0.006 & 6.5 & 0.002 & 2.1 & 16 & Giru? & 0.015 & 5.3 & 0.012 & 4.1 \\
\hline
\end{tabular}

${ }^{a}$ Fluxes are scaled relative to that of Loki. 
right image in Figure 6 shows the projected face of Io as seen from Earth at the time of the observations based on the United States Geological Survey (USGS) Geological Map. ${ }^{19}$ Known volcanic hot spots are indicated with red triangles. Those features detected at a 5- $\sigma$ confidence in either reconstruction and which also correlate with known hots spots are circled in green and assigned a number. Two prominent sources, numbers 15 and 16 , appear misaligned relative to known hot spots. This may be due to an artifact of the deconvolution process near the limbs of Io over a range of orbital projections that consist of multiple nearby sources.

Table 2 presents the background-subtracted fluxes of each source relative to that of Loki along with the $\mathrm{S} / \mathrm{N}$ from each reconstruction method. Because of its extended nature, the total flux for Loki was determined by summing pixels within a circle with a radius equal to $1.5 \lambda / D$, where $\lambda=4.8 \mu \mathrm{m}$ and $D=22.8$ meters. The background was subtracted using a 1-pixel wide annulus immediately outside the integrated circle. For the remaining point-like sources, the circular radius was reduced to $\lambda / D$.

\section{CONCLUSIONS}

Employing 'lucky Fizeau' imaging at $M$-Band, we demonstrate the ability of the LBT to successfully obtain diffraction-limited interferometric images using LBTI/LMIRcam. As Io rotated on the sky with respect to the changing parallactic angle, the PSF's interferometric fringe pattern probed varying angular positions, increasing coverage of the uv plane. Reconstruction of the composite images enabled recovery of a spatial resolution resolution equivalent to a 22.8 -meter aperture over a PA range of $-30.0^{\circ}$ to $29.1^{\circ}$, realizing the LBT as the first ELT.

The reconstructed images reveal approximately sixteen independent sources that correspond to known hot spots on the surface of Io. These sources are only recovered after image reconstruction of the Fizeau interferometry and are inaccessible to $M$-Band resolutions typical of 8-meter class telescopes at this wavelength. While shorter near-infrared wavelengths observed using an 10-meter telescope can obtain similar resolutions, longer wavelength observations of Io benefit from the high-temperature emission of the volcanic hot spots as well as lower intensity of scattered light from the Sun.

Future efforts on the current dataset will concentrate on removing deconvolution artifacts and obtain precise flux measurements of the thermal radiation at the hot spot locations. These improvements include de-projecting the current images onto a sphere and synthetically rotating the sphere to a common orientation such that all point sources are properly aligned, which may help minimize ringing in the reconstructed images. In addition, forward modeling of Io based on the USGS Geological Map may further constrain the fluxes from the known volcanic hot spots.

\section{ACKNOWLEDGMENTS}

The LBT is an international collaboration among institutions in the United States, Italy and Germany. LBT Corporation partners are: The University of Arizona on behalf of the Arizona university system; Istituto Nazionale di Astrofisica, Italy; LBT Beteiligungsgesellschaft, Germany, representing the Max-Planck Society, the Astrophysical Institute Potsdam, and Heidelberg University; The Ohio State University, and The Research Corporation, on behalf of The University of Notre Dame, University of Minnesota and University of Virginia.

\section{REFERENCES}

[1] Hinz, P. M., Bippert-Plymate, T., Breuninger, A., Connors, T., Duffy, B., Esposito, S., Hoffmann, W., Kim, J., Kraus, J., McMahon, T., Montoya, M., Nash, R., Durney, O., Solheid, E., Tozzi, A., and Vaitheeswaran, V., "Status of the LBT interferometer," Proc. SPIE 7013 (2008).

[2] Hinz, P. M., Arbo, P., Bailey, V., Connors, T., Durney, O., Esposito, S., Hoffmann, W., F., Jones, T. J., Leisenring, J. M., Montoya, M., Nash, R., Nelson, M. J., McMahon, T., Pinna, E., Puglisi, A., Skemer, A., Skrutskie, M. F., and Vaitheeswaran, V., "On-sky testing of the lbt interferometer: steps toward routine ao-stabilized interferometric observations," Proc. SPIE 8445 (2012). 
[3] Esposito, S., Riccardi, A., Pinna, E., Puglisi, A., Quirós-Pacheco, F., Arcidiacono, C., Xompero, M., Briguglio, R., Agapito, G., Busoni, L., Fini, L., Argomedo, J., Gherardi, A., Brusa, G., Miller, D., Guerra, J. C., Stefanini, P., and Salinari, P., "Large Binocular Telescope Adaptive Optics System: new achievements and perspectives in adaptive optics," Proc. SPIE 8149 (2011).

[4] Bailey, V. P., Hinz, P. M., Vaitheeswaran, V., Puglisi, A., and Esposito, S., "Large Binocular telescope interferometer adaptive optics (LBTIAO): on-sky performance and lessons learned," Proc. SPIE 9148 (2014).

[5] Wilson, J. C., Hinz, P. M., Skrutskie, M. F., Jones, T., Solheid, E., Leisenring, J., Garnavich, P., Kenworthy, M., Nelson, M. J., and Woodward, C. E., "LMIRcam: an L/M-band imager for the LBT combined focus," Proc. SPIE $\mathbf{7 0 1 3}$ (2008).

[6] Skrutskie, M. F., Jones, T., Hinz, P. M., Garnavic, P., Wilson, J. C., Nelson, M. J., Solheid, E., Durney, O., Hoffmann, W. F., Vaitheeswaran, V., McMahon, T., Leisenring, J., and Wong, A., "LMIRcam: an L/M-band imager for the LBT combined focus," Proc. SPIE 7735 (2010).

[7] Leisenring, J. M., Skrutskie, M. F., Hinz, P. M., Skemer, A., Bailey, V., Eisner, J., Garnavich, P., Hoffmann, W. F., Jones, T., Kenworthy, M., Kuzmenko, P., Meyer, M., Nelson, M., Rodigas, T. J., Wilson, J. C., and Vaitheeswaran, V., "On-sky operations and performance of LMIRcam at the Large Binocular Telescope," Proc. SPIE 8446 (2012).

[8] Defrère, D., Hinz, P. M., Downey, E. C., Hill, J. M., Mennesson, B., Skemer, A. J., Vaz, A., Ashby, D. S., Bailey, V. P., Zappellini, G. B., Christou, J. C., Danchi, W. C., Grenz, P., Hoffmann, W., Leisenring, J. M., McMahon, T., Millan-Gabet, R., Montoya, M., and Vaitheeswaran, V., "Co-phasing the Large Binocular telescope: status and performance of LBTI/PHASEcam," Proc. SPIE 9146 (2014).

[9] Herbst, T. M., Ragazzoni, R., Eckart, A., and Weigelt, G., "The LINC-NIRVANA interferometric imager for the Large Binocular Telescope," Proc. SPIE 5492 (2004).

[10] Herbst, T. M., Ragazzoni, R., Eckart, A., and Weigelt, G., "The LINC-NIRVANA Fizeau interferometric imager: final lab integration, First Light experiments and challenges," Proc. SPIE 9146 (2014).

[11] Hofmann, K.-H. and Weigelt, G., "Iterative image reconstruction from the bispectrum," $A \& A$ 278, 328-339 (Oct. 1993).

[12] Hofmann, K.-H., Driebe, T., Heininger, M., Schertl, D., and Weigelt, G., "Reconstruction of aperturesynthesis images from LBT LINC-NIRVANA data using the Richardson-Lucy and space-variant Building Block method," A\& A 444, 983-993 (Dec. 2005).

[13] La Camera, A., Carbillet, M., Olivieri, C., Boccacci, P., and Bertero, M., "AIRY: a complete tool for the simulation and the reconstruction of astronomical images," Proc. SPIE 8445 (July 2012).

[14] Carbillet, M., La Camera, A., Deguignet, J., Prato, M., Aristidi, E., Bertero, M., and Boccacci, P., "Strehlconstrained reconstruction of post-adaptive optics data by means of the Software Package AIRY, version 6.1," Proc. SPIE 9148 (2014).

[15] Bertero, M. and Boccacci, P., "Image restoration methods for the Large Binocular Telescope (LBT)," A\&\&A, Supplement 147, 323-333 (Dec. 2000).

[16] Anconelli, B., Bertero, M., Boccacci, P., Carbillet, M., and Lanteri, H., "Reduction of boundary effects in multiple image deconvolution with an application to LBT LINC-NIRVANA," A\&BA 448, 1217-1224 (Mar. 2006).

[17] Geman, S. and Geman, D., "Stochastic relaxation, Gibbs distributions, and the Bayesian restoration of images," IEEE Trans. Pattern Anal. Mach. Intell. 6, 721-741 (1984).

[18] Bertero, M., Boccacci, P., La Camera, A., Olivieri, C., and Carbillet, M., "Imaging with LINC-NIRVANA, the Fizeau interferometer of the Large Binocular Telescope: state of the art and open problems," Inverse Problems 27, 113001 (Nov. 2011).

[19] Williams, D. A., Keszthelyi, L. P., Crown, D. A., Yff, J. A., Jaeger, W. L., Schenk, P. M., Geissler, P. E., and Becker, T. L., "Geologic map of Io: U.S. Geological Survey Scientific Investigations Map 3168, scale 1:15,000,000," (2011). 